Artículo Original

\title{
El género Metacrisia Hampson (Lepidoptera: Erebidae: Arctiinae: Phaegopterina) en los Andes peruanos, con la descripción de una nueva subespecie y especie
}

The genus Metacrisia Hampson (Lepidoptera: Erebidae: Arctiinae: Phaegopterina) in the Peruvian Andes, with the description of a new subspecies and species

$$
\text { Juan Grados }^{1,3} \text {, Georgette Ancajima }^{(\mathbb{D}} \text { y Evelyn Gamboa }{ }^{1}
$$

${ }^{1}$ Museo de Historia Natural, Departamento de Entomología, Universidad Nacional Mayor de San Marcos. Código postal, 15072. 遈 gradosjuan@hotmail.com

${ }^{2}$ Museo de Zoología, Universidade de São Paulo, Avenida Nazaré 481, Ipiranga, 04263-000 São Paulo, SP, Brazil.

${ }^{3}$ Bosque Llaqta, Av. Confraternidad Internacional Este $N^{o} 364$, Huaraz, Perú.

\section{ZooBank: urn:lsid:zoobank.org:pub: 2AB89EB1-BA14-4E9D-A5E3-2582A18E166F https: / / doi.org/10.35249/ rche.46.3.20.12}

Resumen. Se reporta por primera vez el género Metacrisia Hampson en Perú, con la especie Metacrisia courregesi (Dognin), que ocurre en la parte norte de la vertiente occidental de los Andes. Se describen una nueva subespecie, $M$. courregesi mutabilis Grados, ssp. nov. de la Cordillera Blanca (departamento de Huaraz, Perú), y una nueva especie, M. eckstromi Grados, sp. nov., de la selva central de Perú.

Palabras clave: Neotrópico, taxonomía, vertiente occidental de los Andes.

Abstract. The genus Metacrisia Hampson is reported for the first time in Peru; the species Metacrisia courregesi (Dognin) which occurs in the northern part of the western slope of the Andes is newly recorded. The new subspecies $M$. courregesi mutabilis Grados, nov. ssp. from the Cordillera Blanca (Huaraz Department), and the new species M. eckstromi Grados, nov. sp. from the central cloud forest of Peru are described.

Key words: Neotropical, taxonomy, western slope of the Andes.

\section{Introducción}

La cordillera de los Andes es la principal cadena de montañas de Sudamérica, con una extensión aproximada de $9000 \mathrm{~km}$ desde Venezuela hasta el extremo sur de Chile, está ubicada en la parte occidental, teniendo la más alta elevación en la montaña del Aconcagua $(6962 \mathrm{~m})$. El levantamiento de los Andes ha sido uno de los eventos geológicos más importantes, llevado a cabo desde el Mesozoico, en diferentes procesos y distanciados temporalmente, desencadenando un evento vicariante (Garreaud 2009; Graham 2009). Su orogénesis ha tenido un rol preponderante en la variación del clima, en el efecto vicariante marcado en sus lados este y oeste, ha formado diferentes gradientes

Recibido 27 Junio 2020 / Aceptado 12 Agosto 2020 / Publicado online 28 Agosto 2020

Editor Responsable: José Mondaca E. 
altitudinales que derivó en diferentes tipos de hábitats, desde bosques montanos lluviosos, páramos, punas, desiertos y picos glaciales, donde en cada uno de ellos en procesos largos y dinámicos los organismos se han adaptado mediante diferentes procesos evolutivos.

Según nuestro conocimiento actual sobre Arctiinae, algunos géneros están restringidos geográficamente a las partes altas de los Andes, entre los que podemos mencionar a Hypomolis Hampson, Chlorhoda Hampson, Paleomolis Hampson y la mayoría de las especies del género Amastus Walker, dentro de la subtribu Arctiina; Epectaptera Hampson y probablemente todas las especies de Eupyra Herrich-Schäffer en los Ctenuchina; y Chrysocale Walker en los Euchromiina.

El género Metacrisia fue propuesto por Hampson (1901), tomando como especie tipo a Purius courregesi, descrita por Dognin (1891), basado en tres machos y una hembra provenientes de Loja (Ecuador). Actualmente el género consta de tres especies: $M$. courregesi (Dognin, 1901), M. schausi Dognin, 1911 y M. woolfsonae Toulgoët, 1988, las cuales ocurren en los Andes de Colombia y Ecuador. Gaede (1923) describió la especie Metacrisia albida Gaede de Colombia, actualmente considerada dentro del género Elysius Walker y sinonimizada con Elysius melaleuca (Felder) por Vincent y Laguerre (2014).

El poco conocimiento de los Arctiinae altoandinos y sus distribuciones geográficas motivó llevar a cabo el presente trabajo. Se reporta por primera vez para Perú la especie M. courregesi (Dognin), se proporciona la redescripción de $M$. courregesi, se describe una nueva especie y subespecie, y se entrega un mapa con la distribución geográfica de los taxa.

\section{Materiales y Métodos}

Los especímenes estudiados han sido recolectados en varios viajes a la parte norte de Perú, en la vertiente occidental de los Andes y, en un estudio en el Parque Nacional Yanachaga-Chemillén (departamento de Pasco); otros especímenes fueron obtenidos en la visita de Benoit Vincent a Perú. Los especímenes han sido recolectados con trampa de luz, utilizando lámparas de vapor de mercurio y de luz mixta, utilizando frascos letales con acetato de butilo. Especímenes depositados y preservados de las siguientes colecciones fueron examinados:

MNHP Muséum National d'Histoire Naturelle, París, Francia.

MUSM Museo de Historia Natural, Universidad Nacional Mayor de San Marcos, Lima, Perú. USNM National Museum of Natural History, Smithsonian Institution, Washington DC, USA.

Se usó la terminología de la venación alar de acuerdo con Comstock y Needman (1898, 1899), Miller (1970), Wootton (1979) y Common (1990); para las estructuras genitales se siguió a Sibatani et al. (1954), Kuznetsov (1967) y Klots (1970). Los genitales de los especímenes fueron disectados y preparados utilizando una solución de $\mathrm{KOH}(10 \%)$ en baño María. Se utilizó negro de clorazol como medio de tinción para mejorar la observación de los caracteres (Cannon 1937, 1941; Carayon 1969). Las fotografías de los adultos fueron tomadas con una cámara Nikon D80.

\section{Resultados}

Se reporta por primera vez en Perú la especie Metacrisia courregesi courregesi (Dognin, 1891), se describen la nueva subespecie Metacrisia courregesi mutabilis Grados, ssp. nov. de la Cordillera Blanca (Huaraz) en los Andes centrales y la nueva especie Metacrisia eckstromi Grados, sp. nov. de los bosques montanos altos de la selva central de Perú. 


\section{Metacrisia courregesi courregesi (Dognin, 1891)}

(Figs. 1-8)

Diagnosis. Se caracteriza por presentar en el ala anterior, mancha basal, banda basal y postmedial muy bien definidas; la banda postmedial con las manchas irregulares comprende desde el margen costal al margen posterior. Se diferencia de la subespecie $M$. courregesi mutabilis, porque en ésta los caracteres aludidos no son muy definidos.

Redescripción del macho (Figs. 1-2). Cabeza: Proboscis marrón claro. Palpo labial corto y prorecto: primer palpómero rojizo en su área ectal y escamas marrones piliformes largas en la parte ventral; segundo, lado ectal con una mancha azulada en la base y rojizo en la parte distal; lado mesal con escamas piliformes ocráceas; lado ventral, en la base escamas piliformes ocráceas y, amarillentas en la distal; tercero marrón con tinción azulado. Frontoclypeus marrón. Vertex y occiput cremosos. Antena ocrácea. Rami medios cuatro veces el ancho del eje del flagelo. Rami distales disminuyen de tamaño hacia el extremo distal; último ramus bifurcado. Tórax: Patagium cremoso y una línea marrón en la parte mesal. Tegula cremosa en el lado ectal y marrón en la mesal. Parte dorsal del tórax marrón, pleura ocrácea. Patas: Ocráceas. Coxa anterior marrón con ligero tinte morado. Fémures rojizos en sus áreas anteriores con abundantes escamas piliformes en la parte ventral; tibias con mancha marrón en sus partes proximales y rojizo en sus áreas anteriores. Tarsos marrones en sus mitades proximales y con escamas rojizas en las distales. Ala anterior: Longitud ala anterior (19-20 mm) $(\mathrm{n}=8)$. Dorsal: Marrón ocráceo. Mancha cremosa irregular en la base del ala. Una banda basal, irregular, marrón cremosa; contigua a ésta, una banda cremosa irregular; entre ellas, líneas marrones sinusoides. Mancha cremosa en la celda discal. Banda postmedial desde el margen costal y sin llegar al margen posterior; mancha ocrácea continua sinusoide, a los lados pequeñas manchas cremosas de forma variada. Área distal de la $\mathrm{R}_{4}-\mathrm{R}_{5}$ ocrácea. Cerca de la base de la celda anal, escamas rojizas. Ventral: Cremoso hacia la base, con escamas rojizas. Patrón de manchas dorsales visibles no nítidamente. Retinaculum cremoso con escamas rojizas. Ala posterior. Dorsal: Cremoso y algo translúcido. Escamas rojizas a lo largo de la $\mathrm{Cu}_{2}-1 \mathrm{~A}, 1 \mathrm{~A}-2 \mathrm{~A}$ y celda anal. Ventral: Cremoso, con escamas rojizas como en el lado dorsal. Frenulum marrón. Abdomen: Tergum rojizo. Sternum cremoso. Genital del macho (Figs. 5-8) (genitalia \# JGA-681, MUSM): Lado anterior del tegumen cóncavo, en forma de una "V" invertida. Vinculum poco esclerotizado y sinusoide. Saccus poco desarrollado. Unión del tegumen y uncus algo esclerotizado. Uncus esclerotizado, alargado y digitiforme, con ligera constricción en la parte central; setas a lo largo de los lados; base algo más ancha que la parte posterior del tegumen; parte distal esclerotizada, aguda en forma de gancho; con setas en el lado ventral. Juxta esclerotizada, en forma de arco alargado. Transtilla esclerotizada, en forma de "W", con dos procesos esclerotizados algo piriformes, con presencia de espículas. Valvae rectas, anchas y alargadas, cóncavas en el margen dorsal y, sinusoides en la ventral. Proceso ventral membranoso, algo más corto que el dorsal, con setas dispersas. Proceso dorsal más angosto hacia la parte distal, curvo hacia la parte interna. Vista ventral ligeramente cóncava en la parte media del borde mesal; presencia de setas en todo el margen. Aedeagus alargado, ligeramente cóncavo, con ornamentación diminuta en la parte ventro-distal. Coecum desarrollado. Vesica dorsalmente desarrollada, más pequeña que el aedeagus.

Hembra (Figs. 3-4): Ala anterior. Longitud: 21-23 mm $(n=4)$ : mismas características que el macho a excepción por rami más pequeños, los medios del mismo tamaño que el eje del flagelo. Frenulum con cuatro cerdas.

Distribución. Conocida de Loja (Ecuador). En Perú, en los departamentos de Amazonas (Corosha), Cajamarca (Bosque Monteseco y Bosque de Cachil) y La Libertad (El Alisar) (Fig. 25). 

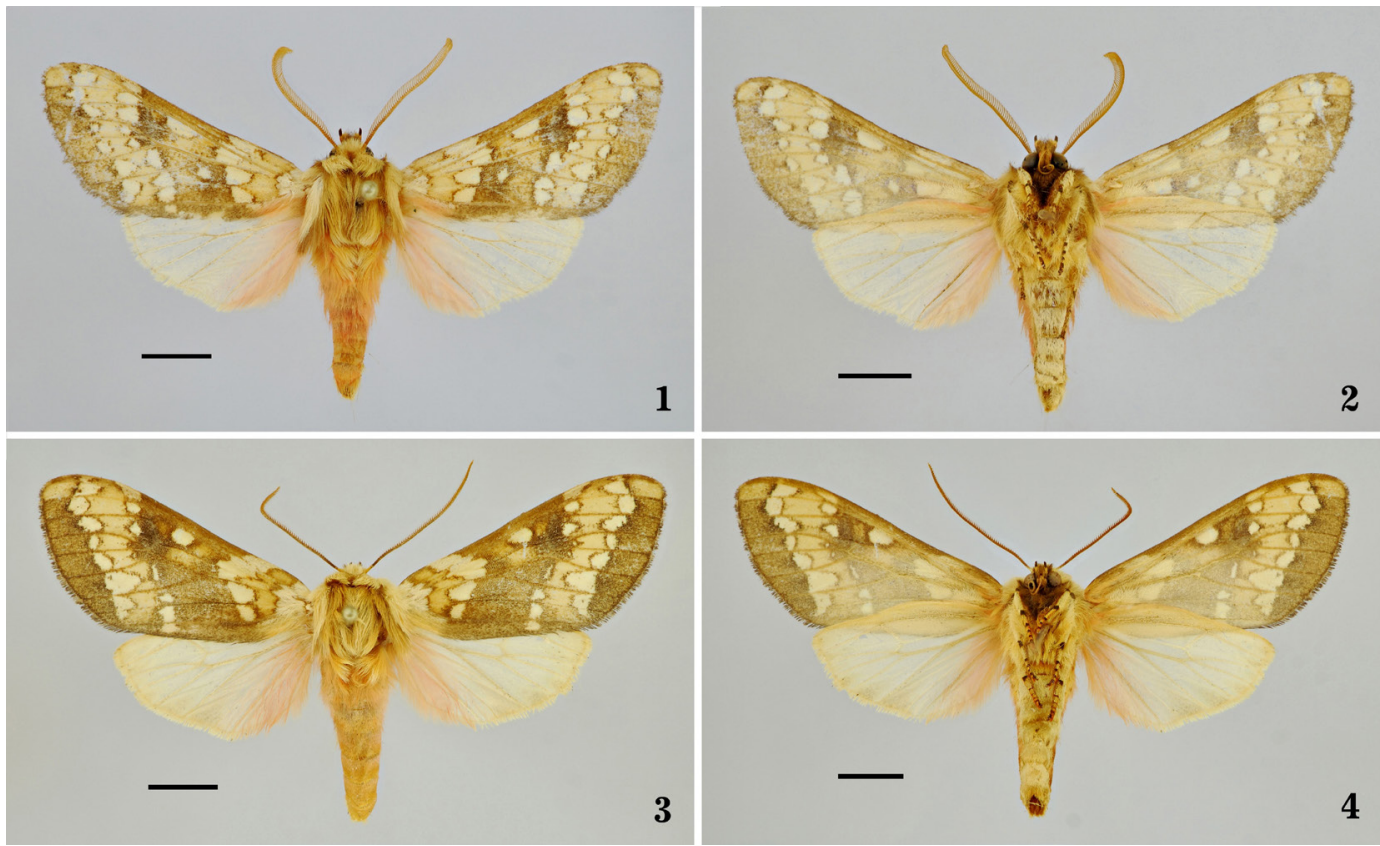

Figuras 1-4. Metacrisia courregesi courregesi (Dognin). 1-2. Macho, vistas dorsal y ventral (Bosque de Cachil). 3-4. Hembra, vistas dorsal y ventral (Bosque de Cachil). Escala: $5 \mathrm{~mm}$.

Comentarios. La especie Metacrisia courregesi (Dognin, 1891) fue descrita en base a tres ejemplares machos y una hembra proveniente de Loja (Ecuador). Tres años después de la descripción original, un ejemplar fue representado en la tercera entrega acerca de los "Lépidoptères de Loja et environs (Equateur)", por Dognin; de acuerdo con el dibujo, correspondería a un ejemplar hembra (Dognin 1894). Watson (1973) presentó fotografías de los genitales del macho, designando a su vez el lectotipo. Los caracteres morfológicos externos de la población del norte de Perú en la vertiente occidental de los Andes coinciden con los caracteres proporcionado por Dognin $(1891,1894)$.
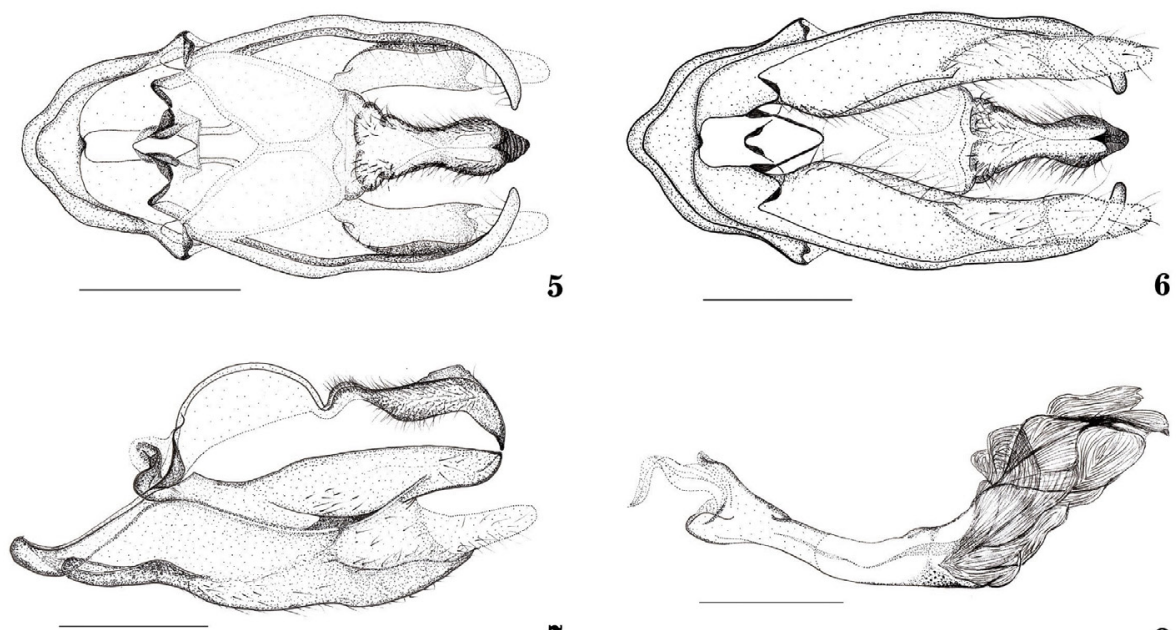

Figuras 5-8. Genital del macho de Metacrisia courregesi courregesi (Dognin). (genitalia \# JGA 426, MUSM). 5-7. Vistas dorsal, ventral y lateral. 8. Aedeagus. Escala: $1 \mathrm{~mm}$. 
Material examinado. PERÚ, Amazonas, 1 macho, Corosha, Bongara (Beirut, at house),

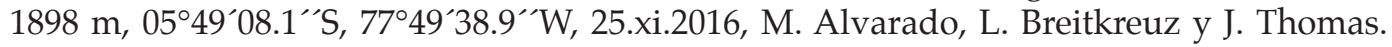
Cajamarca, 1 macho, $4.6 \mathrm{~km}$ al NE de Monteseco, $2841 \mathrm{~m}, 06^{\circ} 50^{\prime} 15.8^{\prime \prime} \mathrm{S}, 7^{\circ} 04^{\prime} 16^{\prime \prime} \mathrm{W}$, 26.iv.2009, J. Grados \& M. Alvarado, L. Figueroa \& E. Bellota (Expedition MUSM-ApecoConservation International); 1 macho, ídem excepto (Genitalia \# JGA - 426, MUSM); 1 macho, ídem excepto (Genitalia \# JGA - 681, MUSM); 1 macho, $5.7 \mathrm{~km}$ al NE de Monteseco, 3116 m, 06 $50^{\circ} 40.4^{\prime \prime}$ S, 7903'45.53”W, 14-15.v.2010, E. Razuri \& C. Carranza (Genitalia \# JGA - 1072, MUSM) (LIGHT TRAP, Mixed Light) (21:00-22:00); 1 hembra, Bosque de Cachil, 2430 m, 07²3’50.9”S, 846’50.3”W, 21.x.2014, J. Grados (LIGHT TRAP, Mixed Light) (21:00 - 22:00); 1 macho, ídem excepto, 23.x.2014 (00:00-01:00); 1 macho, Bosque de Cachil, 2680 m, 07²3’36.57”S, 7846’51.47”W, 22.x.2014, L. Figueroa leg. (LIGHT TRAP, Mixed Light) (22:00-23:00); 2 machos, 1 hembra, ídem excepto, (23:00-00:00); 2 hembras, ídem excepto, (20:00-21:00). La Libertad, 1 hembra, CC El Alisar, 0754’03.96”S, 7808`28.03”'W, 3375 m, 05.x.2014, C. Carranza \& J. Suarez.

\section{Metacrisia courregesi mutabilis Grados, ssp. nov.}

(Figs. 9-18)

Diagnosis. Diferente de la subespecie nominal, porque en el ala anterior las manchas y las bandas no son muy notorias. La subespecie es variable. Algunos ejemplares presentan color ocre claro con las bandas poco perceptibles, otros ejemplares son ocre marrón, presentando algunas manchas pequeñas en la parte postmedial.

Descripción del macho (Figs. 9-14). Ala anterior. Longitud ala anterior (18,5-20 mm) (n = 38). Dorsal: Ocre pálido. Mancha cremosa irregular en la base del ala. Punto cremoso en la celda discal. Las manchas y las bandas en el ala, menos notorias que la especie nominal. La población presenta un rango de variación considerable, pudiéndose en algunos ejemplares observar las bandas y manchas poco perceptibles, mientras que otros presentan el ala marrón ocráceo, con apenas unas manchas claras. Ventral: Celda costal y mitad del área ectal, con tinción azulada. Genital del macho: Con las mismas características de la subespecie nominal.

Hembra (Figs. 15-18): Longitud ala anterior $(22-23 \mathrm{~mm})(\mathrm{n}=2)$. Ocrácea, con la banda postmedial imperceptible. Rami 1,5 veces el ancho del eje del flagelo. Frenulum con cuatro cerdas.

Distribución. Departamento de Ancash, Perú (Fig. 25).

Comentarios. En un estudio de diversidad de Lepidoptera en los bosques de Polylepis en el departamento de Ancash (Cordillera Blanca), se logró capturar una buena serie de ejemplares del género Metacrisia Hampson. Esta población posee un patrón de coloración en las alas muy diferente a las poblaciones de Loja (Ecuador) y del oeste en el norte de Perú. Presenta marcada variación, algunos ejemplares presentan las manchas y bandas, pero poco diferenciadas, mientras otros ejemplares son ocre marrón oscuro. La morfología de los genitales de los machos es similar a M. courregesi.

Etimología. mutabilis es un adjetivo en nominativo singular, que significa, cambiable, variable, inconstante.

Material examinado. Holotipo macho (Figs. 9-10): PERÚ, Ancash, P.N. Huascarán, Entre

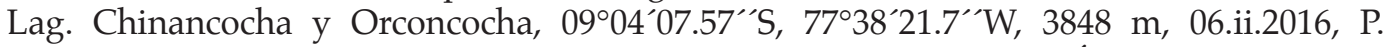
Ancajima \& F. Rodriguez. 33 paratipos (31 machos, 2 hembras). PERÚ, Ancash, 1 macho, 


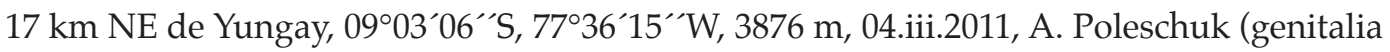
\# JGA - 424, MUSM); 3 machos, P.N. Huascarán, Entre Lag. Chinancocha y Orconcocha, $3848 \mathrm{~m}, 09^{\circ} 04^{\prime} 07.57^{\prime \prime} \mathrm{S}, 7^{\circ} 38^{\prime} 21.7^{\prime \prime} \mathrm{W}, 08 . x \mathrm{.} .2015$, P. Ancajima \& J. Suarez (LIGHT TRAP, Mixed Light) (20:00-21:00); 4 machos, ídem excepto, (21:00-22:00); 1 hembra, ídem excepto, 09.xi.2015 (21:00-22:00); 1 hembra, ídem excepto (22:00-23:00); 1 macho, ídem excepto, 10.xi.2015 (19:00-20:00) (genitalia \# JGA - 482, MUSM); 1 macho, ídem excepto, (21:0022:00) (genitalia \# JGA - 612, MUSM); 1 macho, ídem excepto, 06.ii.2016, P. Ancajima \& F. Rodriguez (21:00-22:00); 1 macho, ídem excepto, 07.ii.2016, (22:00-23:00); 1 macho, ídem excepto, 31.vii.2016, P. Ancajima \& R. Pradel, (22:00-23:00); 2 machos, ídem excepto (21:0022:00); 1 macho, ídem excepto (genitalia \# JGA - 685, MUSM); 1 macho, ídem excepto (23:0000:00); 1 macho, ídem excepto, 1.viii.2016 (22:00-23:00); 1 macho, ídem excepto, 02.viii.2016 (20:00-21:00); 8 machos, 02.viii.2016 (22:00-23:00); 1 macho, ídem excepto (genitalia \# JGA - 683, MUSM); 1 macho, ídem excepto (genitalia \# JGA - 684, MUSM); 2 machos, ídem excepto (23:00-00:00).

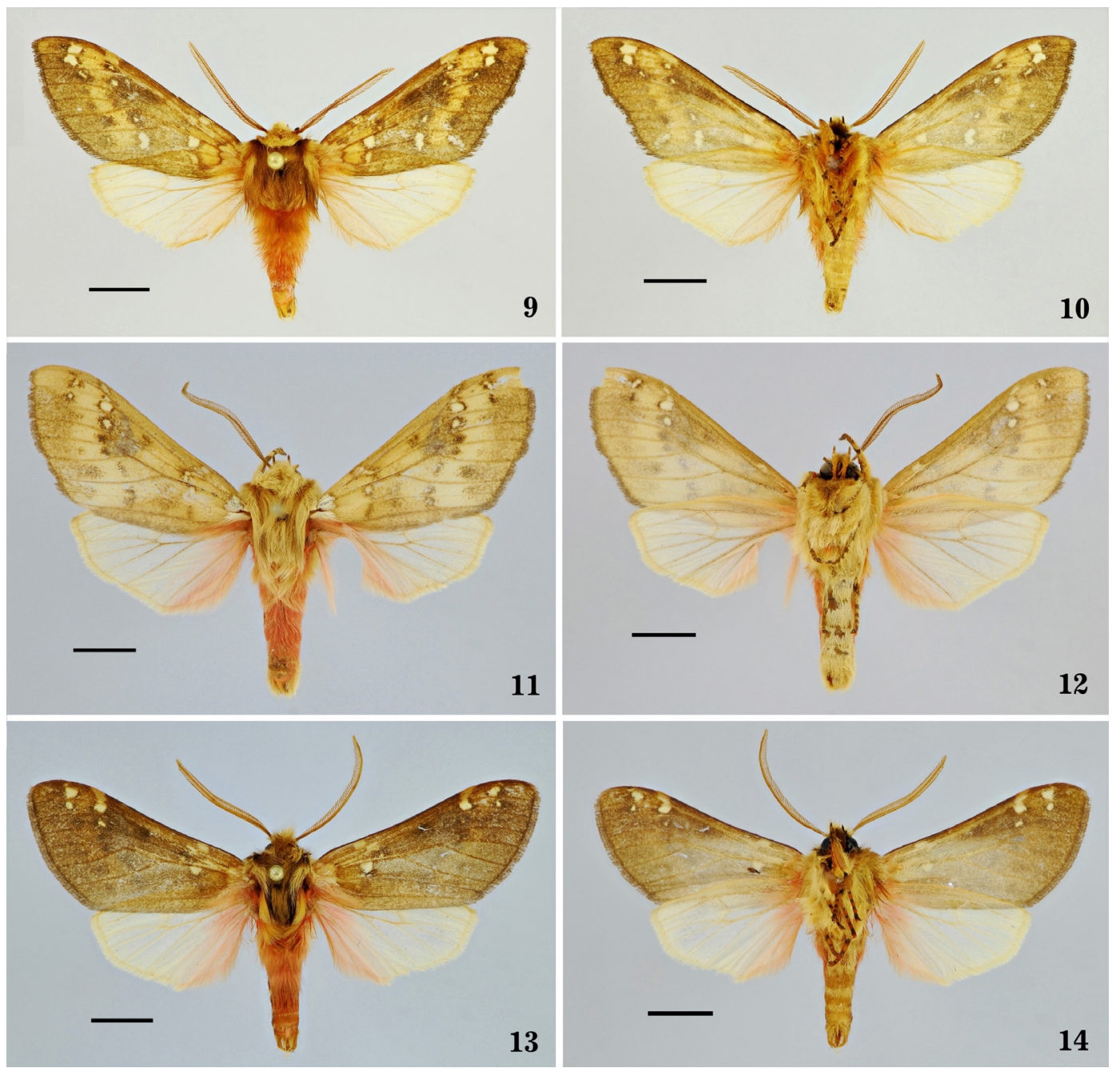

Figuras 9-14. Metacrisia courregesi mutabilis Grados, ssp. nov. 9-10. Holotipo macho, vistas dorsal y ventral. 11-12. Paratipo macho, vistas dorsal y ventral. 13-14. Paratipo macho, vistas dorsal y ventral. Escala: $5 \mathrm{~mm}$. 

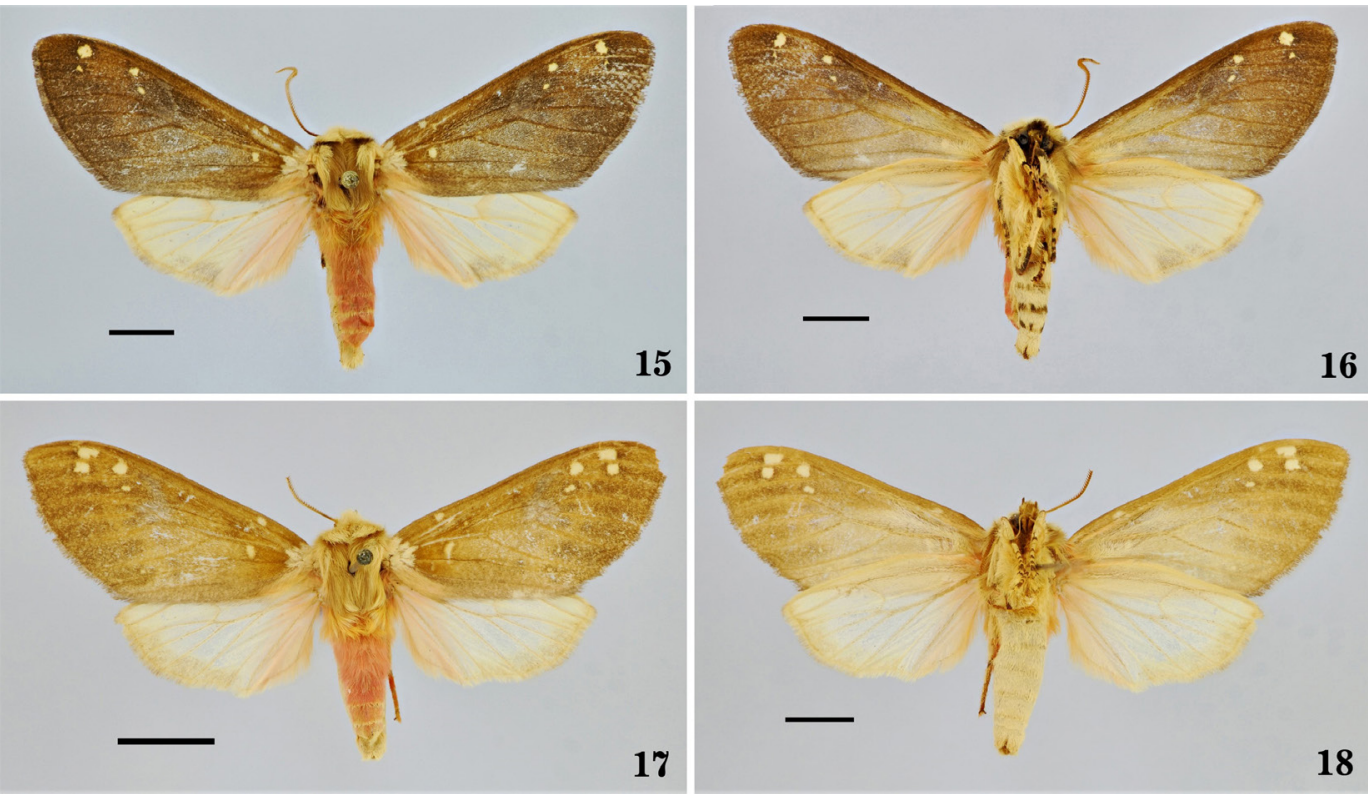

Figuras 15-18. Metacrisia courregesi mutabilis Grados ssp. nov. 15-16. Paratipo hembra, vistas dorsal y ventral. 17-18. Paratipo hembra, vistas dorsal y ventral. Escala: $5 \mathrm{~mm}$.

Metacrisia eckstromi Grados, sp. nov.

(Figs. 19-24)

Diagnosis. Similar a M. c. courregesi, se diferencia porque la banda postmedial es más ancha, llegando casi al termen, mientras que en $M$. c. courregesi, entre la banda postmedial y el termen hay un espacio casi tan ancho como la banda. M. eckstromi presenta el proceso ventral más corto que el proceso dorsal y la vesica es muy corta, mientras en M. c. courregesi el proceso ventral es más largo que el dorsal y, la vesica es tan larga como el aedeagus.

Descripción del macho (Figs. 19-20). Cabeza: Proboscis marrón claro. Palpo labial corto y prorecto: primer palpómero rojo, con escamas piliformes marrón cremoso ventralmente; segundo rojizo; área de la base y la parte ventral con escamas piliformes marrones; tercero marrón. Mitad ventral del frontoclypeus marrón; mitad dorsal del frontoclypeus, vertex y occiput cremosos. Antena marrón claro. Rami medios cuatro veces el ancho del eje del flagelo. Rami distales disminuyen de tamaño hacia el extremo distal; último ramus levemente bifurcado. Tórax: Patagium y tegula cremosos anaranjados; área anterior del patagium con escamas piliformes rojizas; área mesal de la tegula marrón; vista ventral de la tegula: mancha marrón en la parte anterior. Tórax y pleura anaranjados cremosos; mancha marrón sobre el mesoscutellum. Patas: Anaranjadas cremosas. Coxa anterior marrón. Fémures rojizos, en su parte ventral con abundantes escamas piliformes cremosas; mancha marrón en su extremo distal. Tibias con mancha marrón en su parte proximal y rojizo en su área mesal; patas medias y posteriores, la mancha marrón hasta la mitad del largo de la tibia. Tarsos marrones en sus mitades proximales y, escamas rojizas en las distales. Ala anterior: Longitud ala anterior $(20-22 \mathrm{~mm})(\mathrm{n}=8)$. Dorsal: Marrón anaranjado. Parte central de la celda costal y discal, anaranjada. Mancha cremosa irregular en la base del ala. Una banda basal, irregular, marrón anaranjada; contigua a ésta, una banda cremosa irregular formada por tres manchas. Entre ellas, líneas marrones sinusoides. Una mancha cremosa en la celda discal, con borde marrón. Banda postmedial ancha llegando hasta casi el termen, recorre desde el margen costal hasta casi el margen posterior, formado por: 
serie de machas cremosas, y entre éstas una banda continua anaranjada marrón. Una banda angosta marrón entre la banda postmedial y el termen. Escamas rojizas en la base de la celda anal. Ventral: Marrón anaranjado. Mitad basal con escamas rojizas. Patrón de bandas y manchas como el lado dorsal pero tenuemente visibles. Retinaculum cremoso con escamas rojizas. Ala posterior. Dorsal: Cremoso. Escamas rojizas hacia la base y en la celda anal. Ventral: Igual al lado dorsal. Frenulum marrón. Abdomen: Tergum rojizo. Sternum cremoso. Genital del macho: (Figs. 21-24) (genitalia \# JGA-682, MUSM): Lado anterior del tegumen cóncavo, de forma conopial; márgenes posterolaterales sinuosides. Saccus poco desarrollado. Unión del tegumen y uncus algo esclerotizado. Uncus esclerotizado, alargado y digitiforme, con constricción en la parte central; setas a lo largo de los lados; base algo más angosta que la parte posterior del tegumen, invaginación en los dos tercios del largo en su lado dorsal; vista lateral sinusoide, parte distal esclerotizada, aguda en forma de gancho; con setas en los dos tercios proximales, lateral y ventral. Juxta, en vista ventral forma de arco, esclerotizada y más ancha en la parte posterior; vista oblícua, forma de contorno de una " $\mathrm{H}$ ", con los brazos anteriores más anchos. Transtilla esclerotizada, en forma de " $\mathrm{M}$ " de contornos redondeados, con dos procesos esclerotizados posteriores redondeados con presencia de espículas. Valvae rectas, anchas y alargadas, con setas a lo largo del lado ventral, sinusoide en la base del margen ventral y, algo más angosto en la base del margen dorsal. Proceso ventral membranoso, algo más corto que el dorsal, con setas dispersas. Proceso dorsal, más angosto hacia la parte distal, curvo hacia la parte interna. En vista ventral, setas en todo el margen mesal, proceso ventral y dorsal ligeramente curvados hacia el lado interno. Aedeagus corto, con una placa esclerosada en la parte dorsal. Coecum y vesica poco desarrollados.

Hembra. Desconocida.

Etimología. eckstromi es un sustantivo latinizado en genitivo singular. El primer autor y Rainforest Expeditions SAC, quien apoya el proyecto "Discovery new species" en Tambopata, dedican esta nueva especie a Christine Eckstrom, quien junto con su esposo fueron actores claves para crear conciencia y hacer conocida a la región Tambopata en todos los rincones del mundo.

Distribución. En los bosques montanos de la selva central (cordillera de los Andes) de los departamentos de Junín y Pasco (Fig. 25).

Comentarios. Por ahora, solo conocida de los bosques montanos en la vertiente oriental de los Andes de Perú. Así como esta especie, que probablemente es endémica de Perú, las partes medias y altas de la cordillera oriental de los Andes, albergan una alta riqueza de especies que aún está por conocerse.
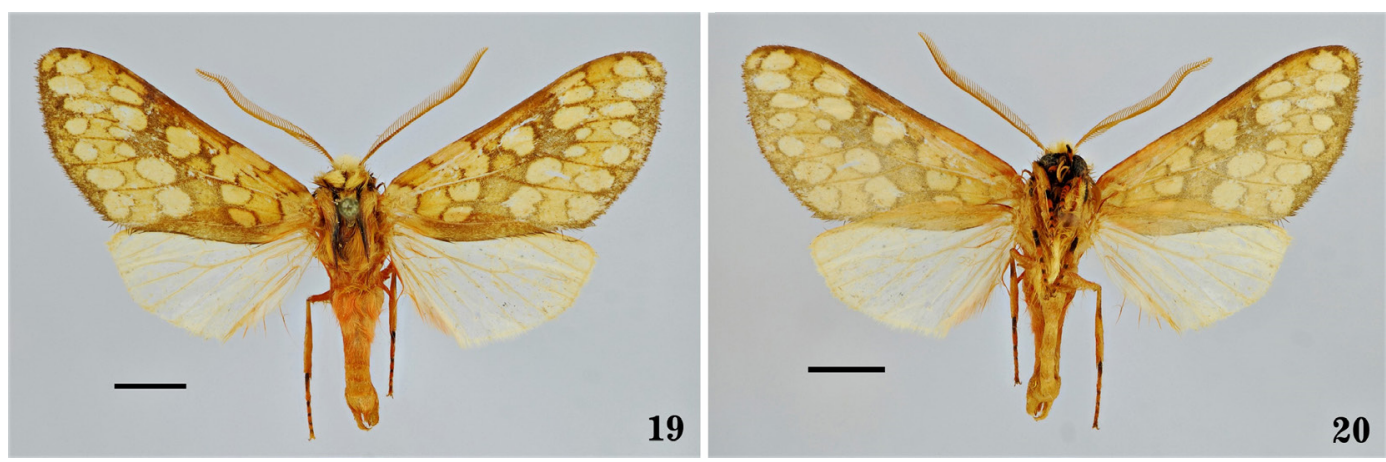

Figuras 19-20. Metacrisia eckstromi Grados, sp. nov. Holotipo macho, vistas dorsal y ventral. Escala: $5 \mathrm{~mm}$. 

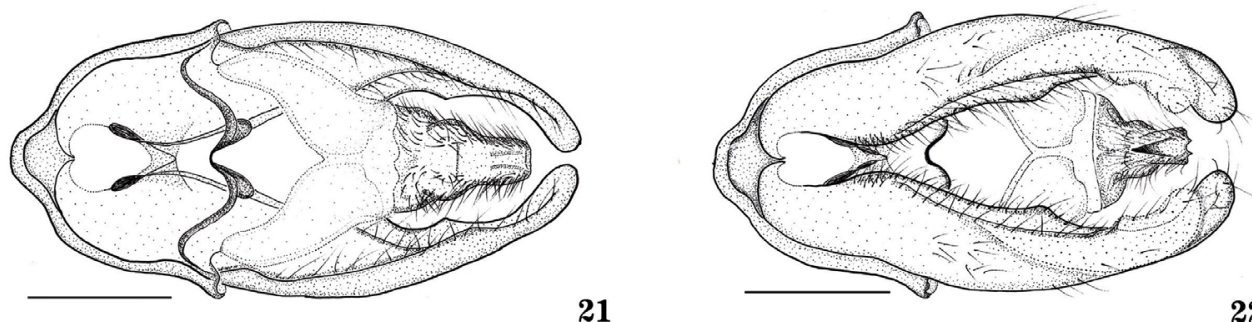

21
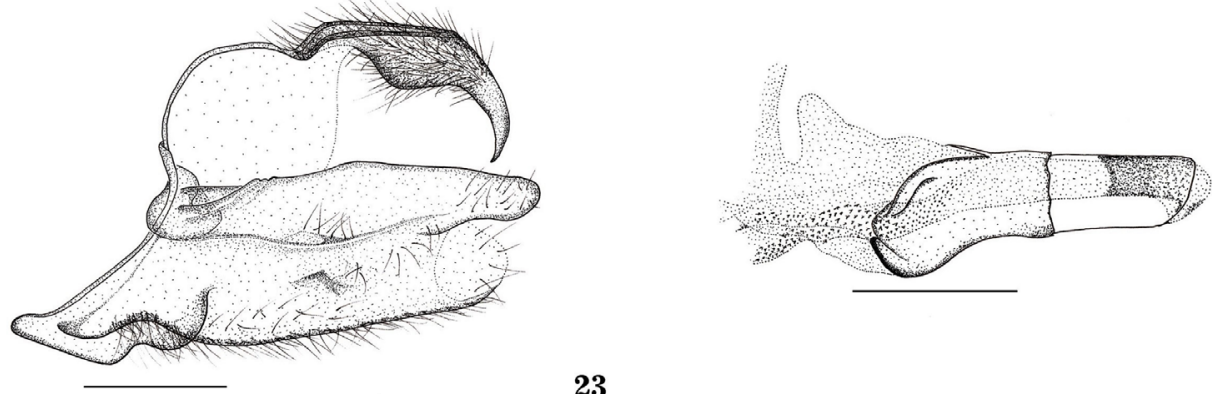

23

Figuras 21-24. Genitalia del macho de Metacrisia eckstromi Grados, sp. nov. (genitalia \# JGA 682, MUSM). 21-23. Vistas dorsal, ventral y lateral. 24. Aedeagus. Escala: $1 \mathrm{~mm}$.

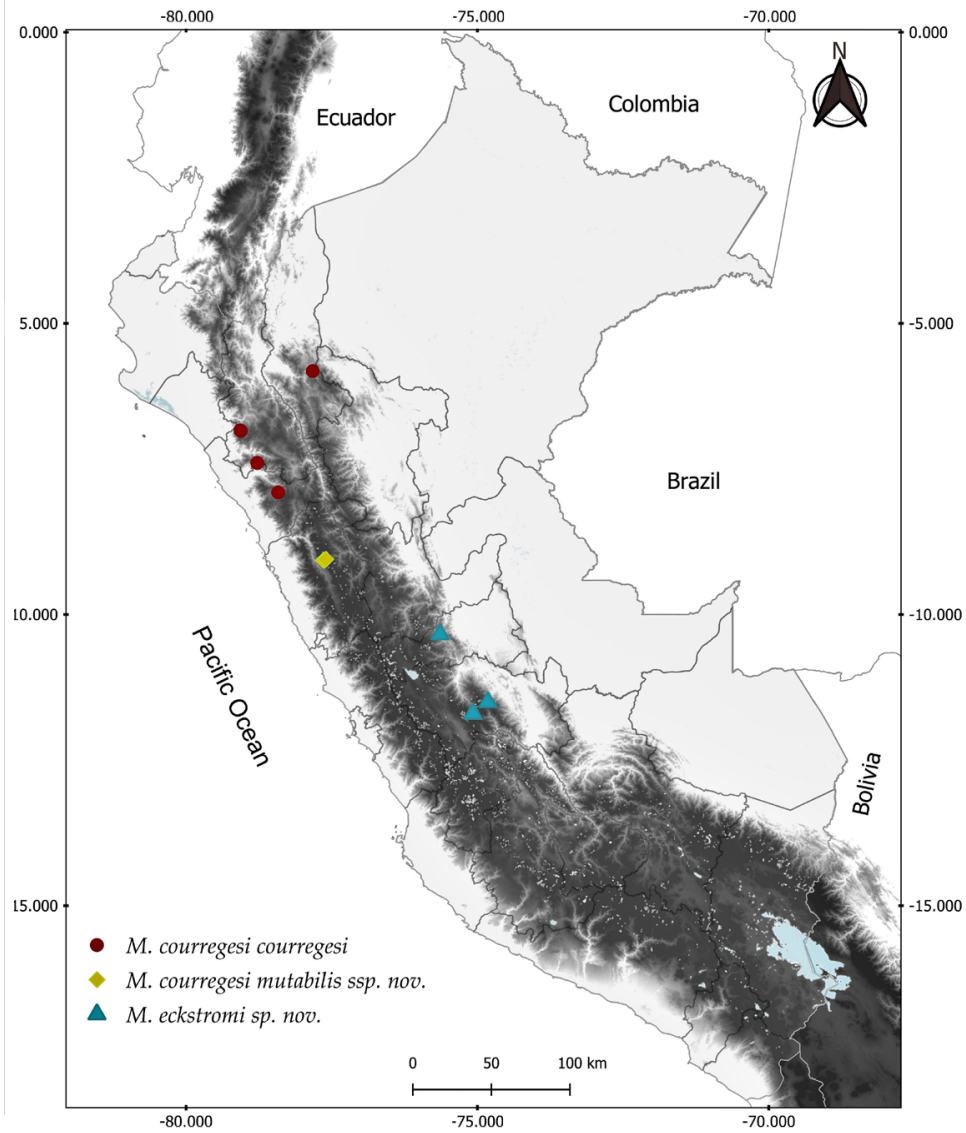

Figura 25. Distribución geográfica del género Metacrisia en Perú. 
Material examinado. Holotipo macho (Figs. 19-20): PERÚ, Pasco, P.N. YanachagaChemillén, ca. Santa Barbara, 10²0’22.7”S, 75³8`27.1”W, 3374 m, 30.viii.2007, J. Grados \& S. Carbonel. 7 paratipos (machos). PERÚ, Junín, 1 macho, Calabaza, $2600 \mathrm{~m}, 11^{\circ} 30^{\prime} 21^{\prime \prime} \mathrm{S}$, 7449’14”W, 30.i.2011, V. Izersky; 1 macho, ídem excepto (genitalia \# JGA - 423, MUSM);

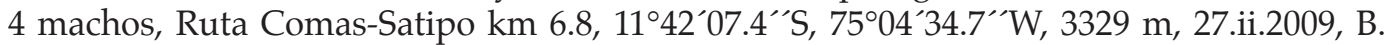
Vincent; 1 macho, ídem excepto (genitalia \# JGA - 682, MUSM).

\section{Agradecimientos}

Queremos agradecer a Conservation International (CI) y a la Asociación Peruana para la Conservación de la Naturaleza (APECO) por la subvención al primer autor, que permitió el estudio de los Arctiinae del bosque nublado Monteseco. A Rainforest Expeditions SAC, por el apoyo con el Proyecto "Discovery new species" en Tambopata. A Benoit Vincent por facilitar algunas muestras que recolectó en su visita a Perú. El viaje al Bosque de Cachil fue subvencionado por la Universidad Nacional Mayor de San Marcos, mediante el proyecto con numeral R.R. 00967-R-14, 141001011. Parte de este trabajo de llevó a cabo bajo el permiso No. 307-2009-AG-SERFOR-DGFFS-DGEFFS proporcionado por la Dirección General Forestal y de Fauna Silvestre - Perú.

\section{Literatura Citada}

Cannon, H.G. (1937) A new biological stain for general purposes. Nature, 139(3517): 549549. https: / / doi.org/10.1038/139549a0

Cannon, H.G. (1941) On Chlorazol black E and some other new stains. Journal of the Royal Microscopical Society, 61: 88-94. https: / / doi.org/10.1111/j.1365-2818.1941.tb00893.x

Carayon, J. (1969) Emploi du noir chlorazol en anatomie microscopique des insects. Annales de la Société Entomologique de France (N.S.), 5(1): 179-193.

Common, I.F.B. (1990) Moths of Australia. Melbourne University Press. 535 pp.

Comstock, J.H. y Needman, J.G. (1898) The wings of insects. American Naturalist, 32(373, $374,376,377,378,380,382,384): 43-48,81-89$, 231-257, 335-340, 413-424, 561-565, 769-777, 903-911.

Comstock, J.H. y Needman, J.G. (1899) The wings of insects. American Naturalist, 33(386, 391, 395): 117-126, 573-582, 843-860. https: / / doi.org/10.1086/ 277462

Dognin, P. (1891) Description de Lépidoptères nouveaux. Le Naturaliste, 2: 257-258.

Dognin, P. (1894) Lépidoptères de Loja et environs (Équateur). Descriptions d'espèces nouvelles. Troisième Livraison, 71-97. http: / / dx.doi.org/10.5962/ bhl.title.9402

Dognin, P. (1911) Hétérocères nouveaux de l'Amérique du Sud. 3, Oberthür \& Rennes, 66 p. http: / / dx.doi.org/10.5962/bhl.title.59881

Gaede, M. (1923) Alte und neue Arctiinae des Berliner Zoologischen Museums. Entomologische Rundschau, 40(6): 23.

Garreaud, R.D. (2009) The Andes climate and weather. Advances in Geosciences, 22: 3-11.

Graham, A. (2009) The Andes: A geological overview from a biological perspective. Annals Missouri Botanical Garden, 96: 371-385.

Hampson, G.F. (1901) Catalogue of the Arctiidae and Agaristidae in the collection of the British Museum 2. London, xx+690 pp.

Klots, A. (1970) Lepidoptera. Taxonomist's Glossary of Genitalia in Insects (ed. Tuxen, S.L.), pp. 97-111. Munksgaard, Copenhagen.

Kuznetsov, N. (1967) Lepidoptera. Fauna of Russia and adjacent countries. Vol. 1. Israel program for Scientific Translations. 305 pp.

Miller, L.D. (1970) Nomenclature of wing veins and cells. Journal of Research on the Lepidoptera, 8(2): 37-48. 
Sibatani, A., Ogata, M., Okada, Y. y Okagaki, H. (1954) Male genitalia of Lepidoptera: Morphology and nomenclature, I. Divisions of the Valvae in Rhopalocera, Phalaenidae (= Noctuidae) and Geometridae. Annals of the Entomological Society of America, 47(1): 93106. https: / / doi.org/10.1093/aesa/47.1.93

Vincent, B. y Laguerre, M. (2014) Catalogue of the Neotropical Arctiini Leach, [1815] (except Ctenuchina Kirby, 1837 and Euchromiina Butler, 1876) (Insecta, Lepidoptera, Erebidae, Arctiinae). Zoosystema, 36(2): 398. http://sciencepress.mnhn.fr/sites/default/files/ articles / pdf / z2014n2a1.pdf

Toulgoët, H. (1988) Description de nouvelles Arctiides d'Amérique latine (Lepidoptera Arctiidae Arctiinae) (28e note). Miscellanea Entomologica, 51(3):79-83.

Watson, A. (1973) An Illustrated Catalog of the Neotropic Arctiinae Types in the United States National Museum (Lepidoptera: Arctiidae). Part II. Smithsonian Contributions to Zoology, 128: 160 pp.

Wootton, R.J. (1979) Function, homology and terminology in insect wings. Systematic Entomology, 4: 81-93. https: / / doi.org/10.1111/j.1365-3113.1979.tb00614.x 